\title{
Central venous pressure measurement is associated with improved outcomes in septic patients: an analysis of the MIMIC-III database
}

\author{
Hui Chen ${ }^{1 \dagger}$, Zhu Zhu ${ }^{2 \dagger}$, Chenyan Zhao ${ }^{1 \dagger}$, Yanxia Guo ${ }^{1}$, Dongyu Chen ${ }^{3}$, Yao Wei ${ }^{1,4^{*}}$ and Jun Jin ${ }^{1,4^{*}}$ (D)
}

\begin{abstract}
Purpose: Measurement of central venous pressure (CVP) can be a useful clinical tool. However, the formal utility of CVP measurement in preventing mortality in septic patients has never been proven.

Methods: The Medical Information Mart for Intensive Care III (MIMIC-III) database was searched to identify septic patients with and without CVP measurements. The primary outcome was 28-day mortality. Multivariate regression was used to elucidate the relationship between CVP measurement and 28-day mortality, and propensity score matching (PSM) and an inverse probability of treatment weighing (IPTW) were employed to validate our findings.

Results: A total of 10,275 patients were included in our study, of which 4516 patients (44\%) underwent CVP measurement within $24 \mathrm{~h}$ of intensive care unit (ICU) admission. The risk of 28-day mortality was reduced in the CVP group (OR 0.60 (95\% Cl 0.51-0.70; $p<0.001)$ ). Patients in the CVP group received more fluid on day 1 and had a shorter duration of mechanical ventilation and vasopressor use, and the reduction in serum lactate was greater than that in the no CVP group. The mediating effect of serum lactate reduction was significant for the whole cohort $(p=0.04$ for the average causal mediation effect (ACME)) and patients in the CVP group with an initial CVP level below $8 \mathrm{mmHg}(p=0.04$ for the ACME).

Conclusion: CVP measurement was associated with decreased risk-adjusted 28-day mortality among patients with sepsis and was proportionally mediated through serum lactate reduction.
\end{abstract}

Keywords: Central venous pressure, Sepsis, Lactate, 28-day mortality

\section{Introduction}

Sepsis is a major challenge in intensive care unit (ICU) settings and accounts for approximately 30 to $50 \%$ of shortterm mortality $[1,2]$. Haemodynamic monitoring plays a critical role in the management of sepsis. As a component of early goal-directed therapies (EGDTs), central venous pressure (CVP) has been utilized to predict status or fluid

\footnotetext{
*Correspondence: dr_betty@126.com; jinjun0514@163.com

${ }^{\dagger}$ Hui Chen, Zhu Zhu and Chenyan Zhao contributed equally to this work.

'Department of Critical Care Medicine, The First Affiliated Hospital of Soochow University, Suzhou 215000, Jiangsu, China

Full list of author information is available at the end of the article
}

responsiveness [3]; however, the most recent guideline no longer recommended CVP to guide fluid administration in septic patients [4]. This statement discounts the physiological value of CVP in clinical. With proper insights, the measurement of CVP can be a useful clinical tool.

Fluid administration is a double-edged sword; the benefits of fluid administration include an increase in cardiac output, but the risks include an increase in hydrostatic pressure, increasing oedema formation. It is more beneficial and less risky to administer fluids in patients with a lower CVP than in those with a high CVP. In a study that included 556 ventilated ICU patients, a

C C The Author(s). 2020 Open Access This article is licensed under a Creative Commons Attribution 4.0 International License, which permits use, sharing, adaptation, distribution and reproduction in any medium or format, as long as you give appropriate credit to the original author(s) and the source, provide a link to the Creative Commons licence, and indicate if changes were made. The images or other third party material in this article are included in the article's Creative Commons licence, unless indicated otherwise in a credit line to the material. If material is not included in the article's Creative Commons licence and your intended use is not permitted by statutory regulation or exceeds the permitted use, you will need to obtain permission directly from the copyright holder. To view a copy of this licence, visit http://creativecommons.org/licenses/by/4.0/ The Creative Commons Public Domain Dedication waiver (http://creativecommons.org/publicdomain/zero/1.0/) applies to the data made available in this article, unless otherwise stated in a credit line to the data. 
positive response to fluid was observed when CVP values were less than $6 \mathrm{mmHg}$ but not when values were greater than $15 \mathrm{mmHg}$ [5]. A retrospective study conducted by Legrand declared that a high CVP value within the first $24 \mathrm{~h}$ of admission was associated with the risk of developing new or persistent acute kidney injury (AKI) [6], and limiting CVP in liver surgery is associated with a decreased risk of bleeding and improved perioperative outcomes [7]. Furthermore, the CVP waveform provides information about inspiratory effort, chest wall and right ventricular compliance and the likelihood of cardiac tamponade [8]. There are limited studies focused on CVP measurement and its effect on outcomes. In a prospective randomized controlled trial [9], CVP measurement and oesophageal Doppler ultrasonography shortened the time to being declared medically fit for discharge in patients undergoing proximal femoral fracture repair under general anaesthesia. Another study demonstrated that a goal-directed protocol using CVP, mean arterial pressure (MAP) and urine output values as therapeutic goals improved survival and clinical outcomes in patients with septic shock [10]. However, the formal utility of CVP measurement in predicting mortality in septic patients has never been proven.

The mediating effects of CVP measurement on mortality are equally important. Therapeutic interventions guided by CVP measurement are aimed at providing adequate oxygen availability and revising tissue hypoperfusion [11]. An elevated lactate level in septic patients is a predictor of poor clinical outcome and a biomarker of tissue hypoperfusion, and a reduction in lactate levels seems to be associated with reduced mortality in critically ill patients [12]. Causal mediation analysis (CMA) [13] was used to investigate the mediating effects of lactate reduction on CVP measurement in terms of mortality.

In the present study, we aimed to elucidate the effect of CVP measurement on 28-day mortality in septic patients. We hypothesized that CVP measurement was associated with lower 28-day mortality and proportionally mediated through a reduction in lactate.

\section{Methods}

\section{Study design}

We conducted a retrospective cohort study based on a large US-based database called the Medical Information Mart for Intensive Care III (MIMIC-III) [14]. The MIMIC-III (v1.4) database contains comprehensive and high-quality data of well-defined and characterized ICU patients admitted to ICUs at the Beth Israel Deaconess Medical Center between 2001 and 2012. One author (HC) obtained access to the database and was responsible for data extraction (certification number 27252652). Our study complied with the Reporting of
Studies Conducted using Observational Routinely Collected Health Data (RECORD) statement [15].

\section{Selection of participants}

Patients in the MIMIC-III who fulfilled the definition of sepsis were eligible for inclusion. Sepsis was diagnosed according to the sepsis-3 criteria [16]; in brief, patients with documented or suspected infection and an acute change in total Sequential Organ Failure Assessment (SOFA) score of $\geq 2$ points were considered to have sepsis. Infection was identified from the International Classification of Diseases 9th Edition (ICD-9) code in the MIMIC-III. We excluded patients who were younger than 18 years or who spent less than $24 \mathrm{~h}$ in the ICU. Additionally, we analyzed only the first ICU stay for patients who were admitted to the ICU more than once. Included patients for whom initial CVP measurements were completed within $24 \mathrm{~h}$ after ICU admission were classified as the CVP group, and the rest of the patients comprised the no CVP group.

\section{Variable extraction}

The primary exposure was whether the patients underwent CVP measurements. The time to initial CVP measurement, the initial level of CVP and the duration of use of CVP were also collected. Baseline characteristics within the first $24 \mathrm{~h}$ after ICU admission were collected using structured query language (SQL), including age, sex, weight, ICU type, severity at admission as measured by SOFA score, the Simplified Acute Physiology Score II (SAPS II) and the Elixhauser comorbidity score. The use of mechanical ventilation, application of renal replacement therapy (RRT), and administration of vasopressors were also recorded. Vital signs included the MAP, heart rate, temperature $\left({ }^{\circ} \mathrm{C}\right)$ and respiratory rate. Laboratory variables including white blood cell (WBC) count, haemoglobin, platelet counts, lactate, $\mathrm{pH}$, partial pressure of oxygen $\left(\mathrm{PO}_{2}\right)$ and partial pressure of carbon dioxide $\left(\mathrm{PCO}_{2}\right)$ were measured during the first $24 \mathrm{~h}$ in the ICU. If a variable was recorded more than once in the first $24 \mathrm{~h}$, we used the value related to the greatest severity of illness. The incidence of AKI was also extracted, and AKI was defined according to the Kidney Disease Improving Global Outcomes (KDGIO) criteria.

Comorbidities including congestive heart failure (CHF), atrial fibrillation (AFIB), chronic renal disease, liver disease, chronic obstructive pulmonary disease (COPD), stroke and malignant tumour were also collected for analysis based on the recorded ICD-9 codes in the MIMIC-III database.

\section{Outcomes}

The primary outcome in the present study was 28-day mortality. Secondary outcomes included in-hospital and 
1-year morality; the incidence of AKI within 7 days after ICU admission; the volumes (L) of intravenous fluid (IVF) in the first, second and third days in the ICU; the number of ventilator-free and vasopressor-free days within 28 days after ICU admission; and reduction in serum lactate (calculated as the difference between the maximum lactate level on day 1 and day 3 ).

\section{Statistical analysis}

Values are presented as the means (standard deviations) or medians [interquartile ranges (IQRs)] for continuous variables, and categorical variables are presented as total numbers and percentages. Comparisons between groups were made using the $X^{2}$ test or Fisher's exact test for categorical variables and Student's $t$ test, or the MannWhitney $U$ test for continuous variables, as appropriate.

Multivariate regression was selected to characterize the relationship between CVP measurement and the primary outcome. Baseline variables that were considered clinically relevant or that showed a univariate relationship with the outcome $(p<0.10)$, including age, sex, weight admission period, severity score, use of mechanical ventilation, use of RRT, use of vasopressors, comorbidities, AKI, vital signs (MAP, heart rate, temperature and respiratory rate) and initial lactate level, were entered into a multivariate logistic regression model as covariates. To avoid bias induced by missing data, the analysis of the primary outcome was duplicated after multiple imputations.

Propensity score matching (PSM) and propensity score-based inverse probability of treatment weighing (IPTW) were also used to adjust the covariates to ensure the robustness of our findings [17, 18]. A multivariate logistic regression model was used to estimate the patient's propensity scores for CVP measurement. One-toone nearest neighbour matching with a calliper width of 0.05 was applied in the present study. An IPTW model was created using the estimated propensity scores as weights. The standardized mean differences (SMDs) were calculated to evaluate the effectiveness of the PSM and IPTW. Logistic regression was then performed on the matched cohort and weighted cohort, separately. Outcomes and therapeutic interventions were generated from the matched cohort.

CMA is a method to differentiate the total effect of a treatment into direct and indirect effects. The indirect effect on the outcome is mediated via a mediator. The analysis produces an average causal mediation effect (ACME), average direct effect (ADE) and total effect. To explore whether the effect of CVP measurement on the primary outcome is proportionally mediated by the reduction in serum lactate, we used CMA to characterize the causality relationship in our retrospective study.
Subgroups analyses of patients with positive blood cultures and septic shock were performed. Given that the effect of CVP measurement may vary according to the duration of CVP measurement and the initial CVP level, we first performed a series of multivariate logistic regressions based on the different duration of CVP measurement ( 1 day, 2 days, 3 days, 4 days, 5 days, 6 days, 7 days and $>7$ days), and then conducted sensitivity analyses comparing patients whose initial CVP level was below $8 \mathrm{mmHg}$ or above $15 \mathrm{mmHg}$ with patients in the no CVP group to evaluate the robustness of our findings.

All statistical analyses were performed using RStudio (version 1.2.5019), and $p<0.05$ was considered statistically significant.

\section{Results \\ Baseline characteristics}

After reviewing the data of 18,592 septic patients, a total of 10,275 patients were included in our study. The flow diagram of patient selections is presented in Fig. 1. Among the study cohort, 4516 patients (44\%) had their CVP measured within $24 \mathrm{~h}$ after ICU admission, with a median value of $11 \mathrm{mmHg}$ (IQR, $8-15 \mathrm{mmHg}$ ); the duration of use of CVP was 2.9 days (IQR, 1.3-7.4 days); and the time to initial CVP measurement was $3 \mathrm{~h}$ (IQR, 1.6-7.8 h) (Additional file 1: Fig. S1). The baseline characteristics of the CVP and no CVP groups are summarized in Table 1. Patients in the CVP group had significantly higher SOFA scores (6 (4-9) vs. 4 (3-6)) and lactate levels $(1.9 \mathrm{mmol} / \mathrm{L}$ (IQR, $1.3-3.1 \mathrm{mmol} / \mathrm{L})$ vs. $1.5 \mathrm{mmol} / \mathrm{L}(\mathrm{IQR}, 1.1-2.1 \mathrm{mmol} / \mathrm{L})$ ) on admission than those in the no CVP group. Within the first $24 \mathrm{~h}$ after ICU admission, the CVP group was more likely to receive mechanical ventilation (74.5 vs. $34.2 \%)$ and vasopressors (31.1 vs. $4.3 \%$ ) than the no CVP group.

\section{Primary outcome}

The multivariate logistic regression analyses showed a significant beneficial effect of CVP measurement in terms of 28-day mortality (Fig. 2), with an adjusted odds ratio (OR) of 0.60 (95\% CI $0.51-0.70$ ). The results were maintained after multiple imputations for missing values (Additional file 1: Table S1 and S2). After PSM and IPTW, the imbalance in the covariates between the CVP and no CVP groups was significantly minimized (Additional file 1: Fig. S2), and the association remained robust (Fig. 2). We also performed subgroup analyses to investigate patients with positive blood cultures and septic shock, and both subgroup analyses produced the same result (Additional file 1: Table S3 and S4). 


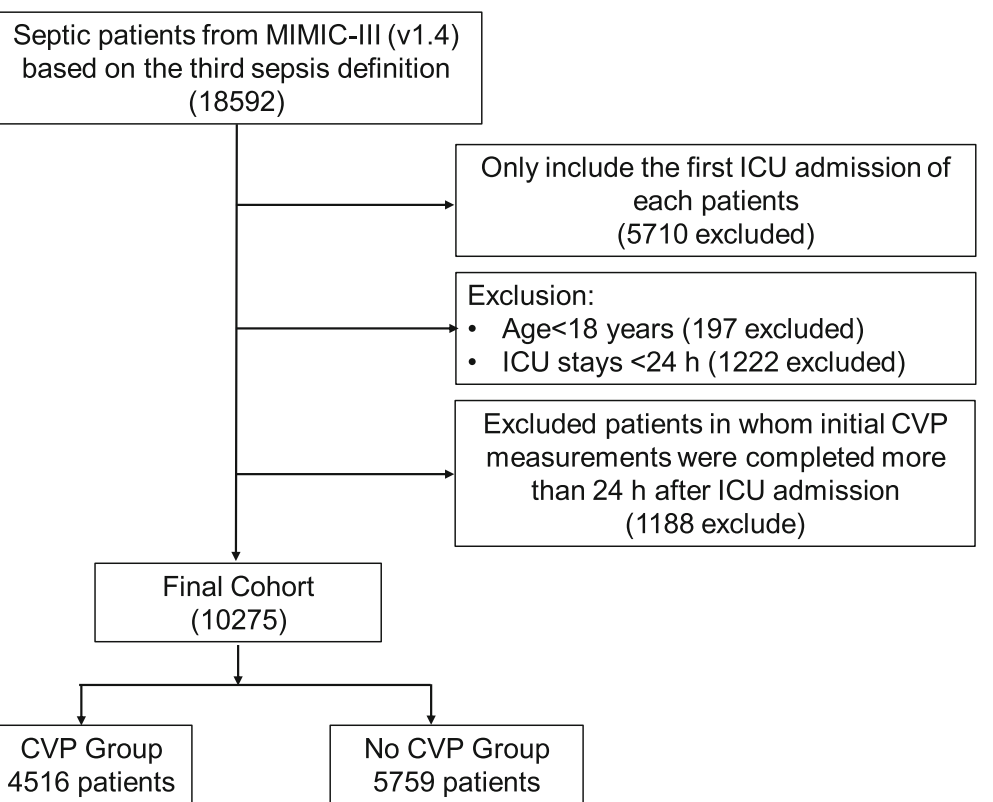

Fig. 1 Study flow diagram in the present study

\section{Secondary outcomes with propensity score-matched cohorts}

CVP measurement was also associated with lower riskadjusted in-hospital mortality and 1-year mortality, but not with AKI within 7 days after ICU admission (Fig. 3). Numerous therapeutic interventions that might account for the beneficial effects of CVP measurement were also investigated. Compared with the no CVP group, the volume of IVF in the CVP group was significantly higher on day 1 ( 2.4 vs. $1.9 \mathrm{~L} ; p<0.001)$, but there were no differences on day 2 and day 3. Patients in the CVP group had shorter durations of mechanical ventilation and vasopressor use than patients in the no CVP group. With respect to lactate, we observed that the reduction in serum lactate between day 1 and day 3 was higher in the CVP group than in the no CVP group (1.48 vs. 1.13 $\mathrm{mmol} / \mathrm{L} ; p=0.029)$. Table 2 shows the detailed results.

CMA showed that the reduction in serum lactate mediated $11 \%(95 \%$ CI $0.7 \%-66 \% ; p=0.04)$ of the beneficial effect of CVP measurement ( $p=0.04$ for ACME) in terms of 28-day mortality in septic patients (Fig. 4).

\section{Sensitivity analyses}

The duration of CVP measurement did not alter the association (Additional file 1: Table S5). Regarding the initial CVP level, we first included patients with an initial CVP level below $8 \mathrm{mmHg}$ in the CVP group and contrasted them against the no CVP group. The beneficial effect of CVP measurement (Additional file 1: Table S6) and the mediating effect of serum lactate reduction ( $p=$ 0.04 for the ACME) were similar to those in the main analysis. In addition, patients in the CVP group received more IVFs on day 1 and day 2 than those in the no CVP group (Additional file 1: Table S7). We then enrolled patients with an initial CVP level above $15 \mathrm{mmHg}$ as the CVP group and reperformed the analyses. Although CVP measurement remained associated with a lower risk of 28-day mortality (Additional file 1: Table S8), the differences in the reduction in lactate $(1.53$ vs. $1.64 \mathrm{mmol} /$ $\mathrm{L} ; p=0.543)$ and the mediation effect of serum lactate reduction ( $p=0.08$ for the ACME) were nonsignificant (Additional file 1: Table S9).

\section{Discussion}

Our study demonstrated that CVP measurement was associated with significantly lower risk-adjusted 28day mortality than no CVP measurement for the first time, as well as lower in-hospital and 1-year mortality, while no association with AKI within 7-days was detected. The mediating effect of serum lactate reduction on CVP measurement in terms of 28-day mortality was noticeable.

Although previous studies produced conflicting evidence concerning the impact of CVP on septic patients $[19,20]$, CVP has been widely used for more than 60 years to guide fluid therapy [21]. Understanding how CVP measurement influences clinicians' decisions has improved over recent years. We abandoned targeting a specific CVP value due to the heterogeneity of patients; rather, we combined the trends of CVP and cardiac output to titrate fluid administration rather than rely on CVP alone. We realized that maintaining CVP as low as 
Table 1 Comparisons of baseline characteristics between the original cohort and matched cohort

\begin{tabular}{|c|c|c|c|c|c|c|}
\hline \multirow[t]{2}{*}{ Covariates } & \multicolumn{3}{|l|}{ Original cohort } & \multicolumn{3}{|l|}{ Matched cohort } \\
\hline & CVP & No CVP & SMD & CVP & No CVP & SMD \\
\hline$N$ & 4516 & 5759 & & 2174 & 2174 & \\
\hline Age & $69(56-79)$ & $71(56-82)$ & 0.079 & $69(56-80)$ & $69(55-81)$ & 0.003 \\
\hline Male (\%) & $2473 / 4516(54.8)$ & 2931/5759 (50.9) & 0.078 & $1131 / 2174(52)$ & 1166/2174 (53.6) & 0.032 \\
\hline Weight (kg) & 79.5 (66.8-95) & $75(62.6-90.2)$ & 0.168 & $78(65-94)$ & $77(64.5-92.5)$ & 0.020 \\
\hline Service unit, $n(\%)$ & & & 0.675 & & & 0.036 \\
\hline MICU & $1811 / 4516(40.1)$ & $3251 / 5759(56.5)$ & & $1113 / 2174(51.2)$ & 1095/2174 (50.4) & \\
\hline SICU/TSICU & $1213 / 4516(26.9)$ & $1451 / 5759(25.2)$ & & 637/2174 (29.3) & $646 / 2174(29.7)$ & \\
\hline CCU/CSRU & 1492/4516 (33.0) & 1057/5759 (18.3) & & $424 / 2174(19.5)$ & $433 / 2174(19.9)$ & \\
\hline Admission period, $n(\%)$ & & & 0.273 & & & 0.004 \\
\hline Before 2008 & $2854 / 4516(63.2)$ & 2868/5759 (49.8) & & 1307/2174 (60.1) & $1311 / 2174(60.3)$ & \\
\hline 2008-2012 & $1662 / 4516(36.8)$ & $2891 / 5759(50.2)$ & & 867/2174 (39.9) & $863 / 2174(39.7)$ & \\
\hline \multicolumn{7}{|l|}{ Severity of illness } \\
\hline SOFA score & $6(4-9)$ & $4(3-6)$ & 0.795 & $5(4-7)$ & $5(3-7)$ & 0.018 \\
\hline SAPS II score & $43(34-54)$ & $37(30-46)$ & 0.465 & $41(32-49)$ & $40(32-50)$ & 0.008 \\
\hline Elixhauser comorbidity score & $7(3-12)$ & $7(2-12)$ & 0.025 & $7(3-13)$ & $7(3-13)$ & 0.013 \\
\hline \multicolumn{7}{|l|}{ Interventions, n (\%) } \\
\hline MV use (1st $24 \mathrm{~h})$ & $3366 / 4516(74.5)$ & 1971/5759 (34.2) & 0.885 & $1189 / 2174(54.7)$ & $1203 / 2174(55.3)$ & 0.013 \\
\hline Vasopressor use (1st 24 h) & 1406/4516 (31.1) & 246/5759 (4.3) & 0.752 & 259/2174 (11.9) & 236/2174 (10.9) & 0.033 \\
\hline RRT use (1st $24 \mathrm{~h})$ & 238/4516 (5.3) & $280 / 5759(4.9)$ & 0.019 & $116 / 2174(5.3)$ & 104/2174 (4.8) & 0.025 \\
\hline \multicolumn{7}{|l|}{ Comorbidities, $n$ (\%) } \\
\hline $\mathrm{CHF}$ & $996 / 4516(22.1)$ & $1296 / 5759(22.5)$ & 0.011 & $535 / 2174(24.6)$ & $551 / 2174(25.3)$ & 0.002 \\
\hline AFIB & $990 / 4516(21.9)$ & 1428/5759 (24.8) & 0.068 & $535 / 2174(24.6)$ & $527 / 2174(24.2)$ & 0.009 \\
\hline Chronic renal disease & $608 / 4526(13.5)$ & 1129/5759 (19.6) & 0.166 & $334 / 2174(15.4)$ & $329 / 2174(15.1)$ & 0.006 \\
\hline Liver disease & 405/4526 (9.0) & $440 / 5759(7.6)$ & 0.048 & 183/2174 (8.4) & 183/2174 (8.4) & $<0.001$ \\
\hline COPD & $908 / 4526(20.1)$ & $1272 / 5759(22.1)$ & 0.049 & $477 / 2174(21.9)$ & 458/2174 (21.1) & 0.021 \\
\hline Stroke & 151/4526 (3.3) & $231 / 5759(4.0)$ & 0.035 & $90 / 2174(4.1)$ & $83 / 2174$ (3.8) & 0.016 \\
\hline Malignancy & 152/4526 (3.4) & 201/5759 (3.5) & 0.007 & 73/2174 (7.4) & $76 / 2174$ (3.5) & 0.008 \\
\hline \multicolumn{7}{|l|}{ Vital signs } \\
\hline MAP $(\mathrm{mmHg})$ & $54(48-60)$ & $58(51-65)$ & 0.358 & $55(49-62)$ & $56(49-63)$ & 0.013 \\
\hline Heart rate (bpm) & $108(94-124)$ & $104(90-119)$ & 0.229 & $107(92-122)$ & $106(93-121)$ & 0.001 \\
\hline Temperature $\left({ }^{\circ} \mathrm{C}\right)$ & $37.7(37.2-38.3)$ & $37.4(37-38)$ & 0.260 & $37.6(37-38.2)$ & $37.6(37-38.2)$ & $<0.001$ \\
\hline Respiratory rate (bpm) & $28(24-33)$ & $27(24-32)$ & 0.102 & $28(24-33)$ & $28(24-32)$ & 0.004 \\
\hline \multicolumn{7}{|l|}{ Laboratory tests } \\
\hline WBC $\left(\times 10^{9} / \mathrm{L}\right)$ & $15.2(10.8-20.8)$ & $12.2(8.7-16.9)$ & 0.253 & $14.5(10.2-19.7)$ & $13.1(9.3-18.2)$ & 0.046 \\
\hline Hemoglobin $\left(\times 10^{12} / \mathrm{L}\right)$ & $9.2(8-10.5)$ & $10.2(8.9-11.6)$ & 0.486 & $9.7(8.5-11)$ & $9.6(8.4-11)$ & 0.030 \\
\hline Platelet $\left(\times 10^{9} / \mathrm{L}\right)$ & $158(105-231)$ & $197(133-270)$ & 0.277 & $186(126-257)$ & $187(118-261)$ & 0.028 \\
\hline Bicarbonate $(\mathrm{mmol} / \mathrm{L})$ & $21(18-23)$ & $23(19-26)$ & 0.435 & $21(18-24)$ & $21(18-24.8)$ & 0.002 \\
\hline Bun (mg/dL) & $26(17-43)$ & $26(17-42)$ & 0.023 & $27(17-44)$ & $26(17-44)$ & 0.004 \\
\hline Creatinine (mg/dL) & $1.3(0.9-2.1)$ & $1.2(0.8-1.9)$ & 0.009 & $1.2(0.9-2.0)$ & $1.2(0.8-2.0)$ & 0.010 \\
\hline Lactate level (mmol/L) & $1.9(1.3-3.1)$ & $1.5(1.1-2.1)$ & 0.419 & $1.6(1.2-2.4)$ & $1.6(1.1-2.4)$ & 0.024 \\
\hline $\mathrm{pH}$ & $7.36(7.32-7.40)$ & $7.40(7.36-7.44)$ & 0.535 & 7.38 (7.33-7.42) & 7.38 (7.34-7.43) & 0.010 \\
\hline $\mathrm{pO}_{2}(\mathrm{mmHg})$ & 131 (98-178) & 109 (84-146) & 0.345 & 117 (90-156) & $117(90-157)$ & 0.040 \\
\hline $\mathrm{pCO}_{2}(\mathrm{mmHg})$ & $39(35-43)$ & 39 (35-44) & 0.132 & 39 (35-44) & $39(34-44)$ & 0.004 \\
\hline
\end{tabular}


Table 1 Comparisons of baseline characteristics between the original cohort and matched cohort (Continued)

\begin{tabular}{|c|c|c|c|c|c|c|}
\hline \multirow[t]{2}{*}{ Covariates } & \multicolumn{3}{|l|}{ Original cohort } & \multicolumn{3}{|l|}{ Matched cohort } \\
\hline & CVP & No CVP & SMD & CVP & No CVP & SMD \\
\hline $\mathrm{AKI}, n(\%)$ & $3488 / 4526(77.2)$ & $3502 / 5759(60.8)$ & 0.361 & $1507 / 2174(69.3)$ & $1489 / 2174(68.5)$ & 0.018 \\
\hline
\end{tabular}

possible after initial haemodynamic stabilization was beneficial. We also extract valid information from CVP waves to aid clinicians [22]. However, the implications of CVP measurement on septic patient outcomes have never been questioned.

In our study, the SOFA score, SAPS II score, lactate level and incidence of AKI were significantly higher in the CVP group than in the no CVP group. Patients in the CVP group received mechanical ventilation and vasopressors more frequently than those in the no CVP group, and the MAP was lower in the CVP group than in the no CVP group. Despite these results, we found notably lower mortality in patients in CVP group than in those in the no CVP group after adjustment for confounding factors, and the relationship was robust regardless of the duration of CVP measurement and the initial CVP level. Consistent with limited results, in a nationwide, 1-day, prospective, point prevalence study, Machado et al. [1] declared that limited resources to treat sepsis (including the reduced availability of CVP measurements) were associated with increased mortality. In conclusion, our results highlight an essential role of
CVP measurement in septic patients, and CVP measurement should not be abandoned at any time.

It was difficult to explore whether the therapeutic interventions were prompted by CVP measurement, and which interventions might account for the beneficial effects of CVP measurement in our retrospective observational study. CMA was applied to cover this limitation, and we hypothesized that CVP measurement-related triggers including fluid therapy could normalize lactate in septic patients with elevated lactate levels and further improve outcomes. In the present study, the volume of IVFs on day 1 and the reduction in serum lactate between day 1 and day 3 were higher in the CVP group than in the no CVP group. We used CVP measurement as the treatment and the reduction in serum lactate between day 1 and day 3 as a mediator variable and found that the effect of CVP measurement on 28-day mortality was proportionally mediated by the reduction in serum lactate.

CVP measurement-related triggers may be influenced by the initial CVP level. Eskesen et al. found a clear CVP level gradient in fluid responsiveness, at $75 \%$ at $0-5$

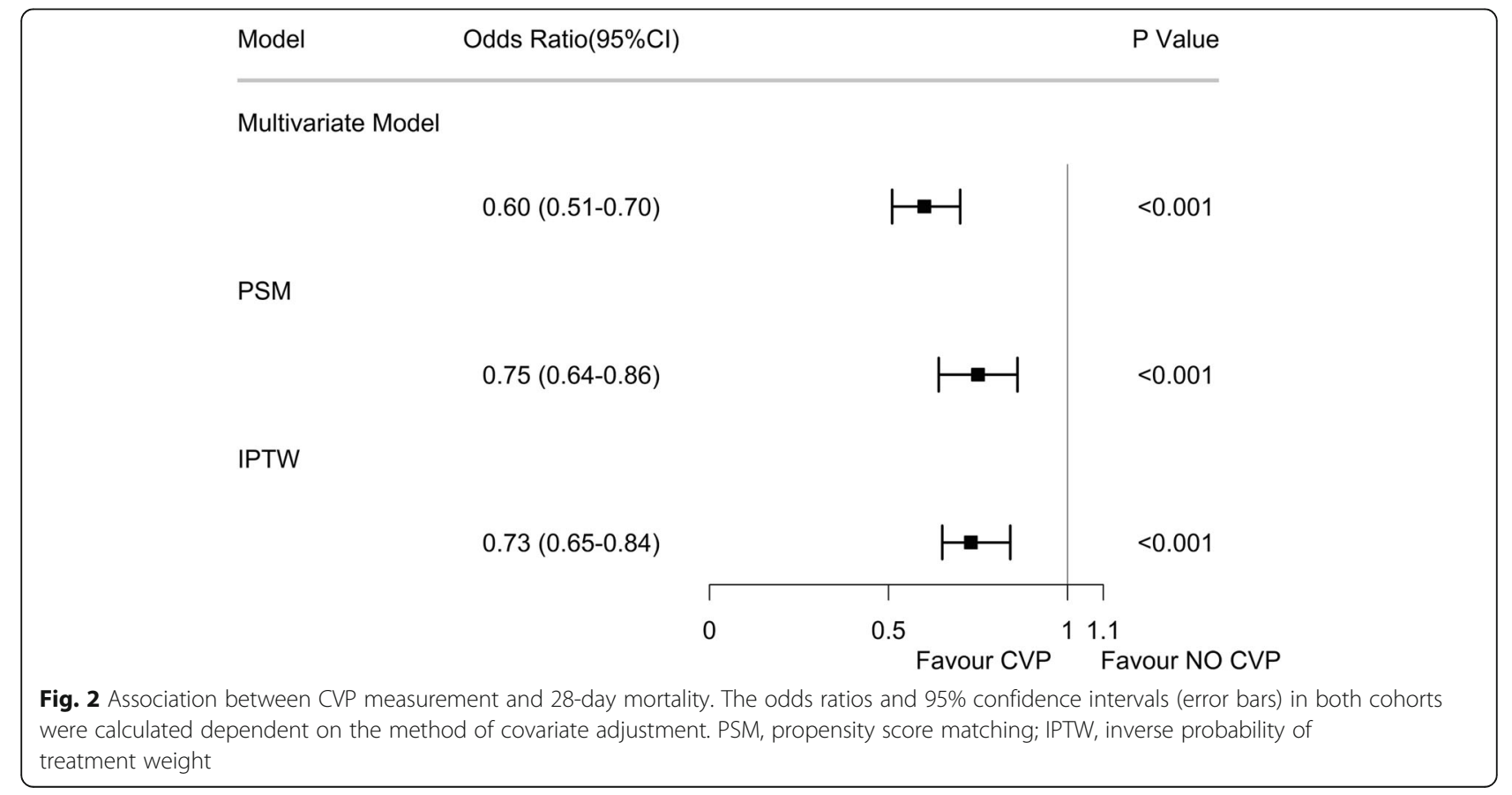




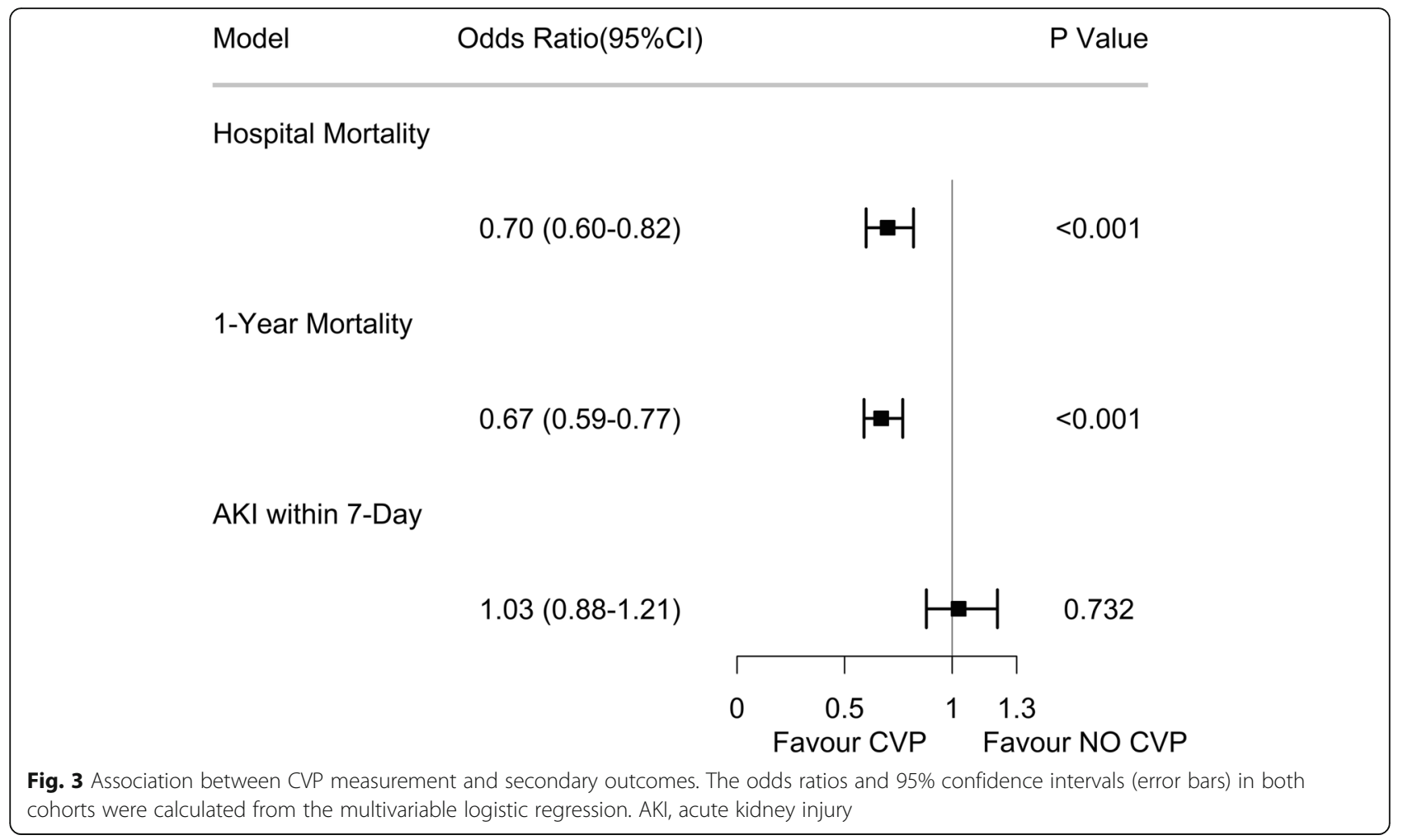

$\mathrm{mmHg}, 55 \%$ at $6-10 \mathrm{mmHg}$, and $15 \%$ from 10 to 14 $\mathrm{mmHg}$, with no patient responding to a level above 13 $\mathrm{mmHg}$ based on 1148 individual data sets [23]. We conducted sensitivity analyses to further understand the impact of the initial CVP level on the association and mediating effect described above. For patients in the CVP group with an initial CVP level below $8 \mathrm{mmHg}$, the volume of IVFs within $48 \mathrm{~h}$ after ICU admission and serum lactate reduction were higher than those in the no CVP group, and the mediating effect of serum lactate reduction was significant. The differences in serum lactate reduction and mediating effects were nonsignificant for patients in the CVP group with an initial CVP level above $15 \mathrm{mmHg}$. In line with these findings, a consensus statement recommended immediate fluid resuscitation in shock states associated with very low levels of preload parameters which including CVP [24]; an "extremely" high CVP level should not be used to predict or guide fluid resuscitation but may be used as a safety endpoint to avoid extrathoracic organ injury [22, 25, 26].

Table 2 Clinical outcomes analysis with propensity score matched cohorts

\begin{tabular}{|c|c|c|c|c|}
\hline Outcomes & CVP & NO CVP & Effect size & $p$ value \\
\hline \multicolumn{5}{|l|}{ Primary outcome } \\
\hline 28-day mortality & 396/2174 (18.2) & $500 / 2174(23)$ & 0.118 & $<0.001$ \\
\hline \multicolumn{5}{|l|}{ Secondary outcomes } \\
\hline In-hospital mortality & $366 / 2174(16.8)$ & $415 / 2174(19.1)$ & 0.059 & 0.058 \\
\hline 1-year mortality & $789 / 2174(36.3)$ & $920 / 2174(42.3)$ & 0.124 & $<0.001$ \\
\hline AKI within 7 days, $n$ (\%) & 1722/2174 (79.2) & 1692/2174 (77.8) & 0.029 & 0.355 \\
\hline Volume of IVF on day $1(\mathrm{~mL})$ & $2380(1037-4245)$ & $1897.5(890-3070)$ & 0.327 & $<0.001$ \\
\hline Volume of IVF on day $2(\mathrm{~mL})$ & $997(289.2-2150)$ & $1000(268.25-1953.6)$ & 0.142 & 0.054 \\
\hline Volume of IVF on day $3(\mathrm{~mL})$ & $605(240-1500)$ & $625(230-1580.5)$ & 0.051 & 0.942 \\
\hline Vasopressor-free day in 28 days & $26.6(25-27.4)$ & $26.2(22.9-27.2)$ & 0.472 & $<0.001$ \\
\hline Ventilation-free day in 28 days & $25.8(22.3-27.1)$ & $23.3(17.3-26.2)$ & 0.291 & $<0.001$ \\
\hline Delta-lactate & $1.48(2.35)$ & $1.13(2.32)$ & 0.148 & 0.029 \\
\hline
\end{tabular}

IVF intravenous fluid, $A K l$ acute kidney injury 


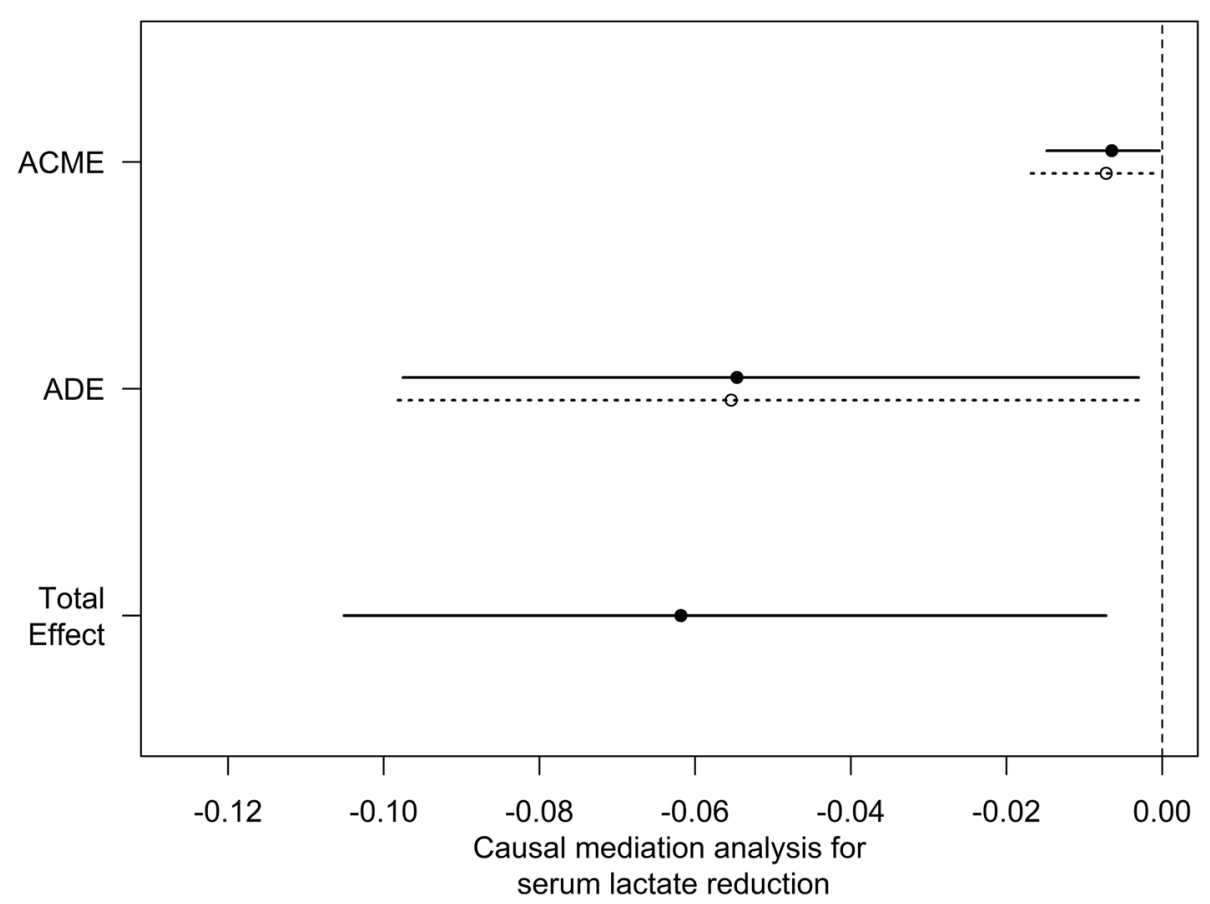

Fig. 4 Causal mediation analysis for serum lactate reduction. The solid line represents the CVP group, and the dashed line represents the no CVP group

However, additional studies are needed to determine which interventions or protective factors mediate the beneficial effect of CVP measurement in patients, especially patients with an "extremely" high CVP level.

Our results simply reflect the true effect of CVP measurement in real-world clinical practice and confirm the benefit of CVP measurement in septic patients regardless of the initial CVP level. Clinicians should not abandon the measurement of CVP but consider how to utilize CVP in an appropriate way.

Several limitations in the present study should be considered. First, the definition of sepsis was based on infection and organ failure, but the infection diagnoses were undefined [27]. Hence, we included septic patients with positive blood cultures results in the subgroup analysis. Second, since the MIMIC-III data ranged from 2001 to 2015 , the versions of the bundles might have changed during the period, and the results may not reflect current practices; hence, our results were adjusted for the admission period; in MIMIC-III database, we could not obtain the exact year of patient admission, and we divided patients into two groups in terms of admission time period (before 2008 and 2008-2012) for enrolment in the model. Third, there were multiple unmeasured confounders in our study that could affect our findings, such as different decisions by clinicians after CVP measurement and interventions before CVP measurement, and the use of other haemodynamic monitoring techniques in each group, including transthoracic echocardiography; pulmonary arterial catheters' placement was unknown. Fourth, our study was a retrospective cohort study based on electronic healthcare records, the data generated during routine clinical visits. There are several technical challenges in measuring CVP, such as zero levels and reading errors, and the reliability of CVP levels in the present study is questionable. Fifth, when conducting the sensitivity analyses, except for the technical challenges, there are lots of factors that influence the initial CVP levels, including the initial PEEP, the expiratory phase when measured and the intraperitoneal pressure [8], and it is difficult to adjust these factors in a retrospective observational study. Finally, the causal relationship between CVP measurement and 28-day mortality was not explored thoroughly, and the reduction in lactate could have nothing to do with CVP measurement [28], as CVP measurementrelated triggers are complex in clinical practice. A randomized study comparing the effect of CVP measurement and no CVP measurement is needed in the future.

\section{Conclusion}

In conclusion, CVP measurement was associated with decreased risk-adjusted 28-day mortality in septic patients. The reduction in serum lactate may have mediated this effect, especially in patients with low CVP levels. In patients with high CVP levels, the mediating effect may be related to the reduced risk of extrathoracic organ injury. 


\section{Supplementary information}

Supplementary information accompanies this paper at https://doi.org/10. 1186/s13054-020-03109-9.

Additional file 1: Table S1. Percentage of missing data in the variables of interest. Table S2. Association between CVP measurements and 28day mortality with different models. Table S3. Analysis for patients with positive blood cultures ( $n=4330$ ). Table S4. Analysis for patients with septic shock $(n=2568)$. Table S5. Sensitivity analyses for patients with different duration of CVP measurements. Table S6. Sensitivity analysis for patients with an initial CVP level below $8 \mathrm{mmHg}$ in CVP group. Table S7. Clinical outcomes after sensitivity analysis (CVP $<8 \mathrm{mmHg})$. Table S8. Sensitivity analysis for patients with an initial CVP level above $15 \mathrm{mmHg}$ in CVP group. Table S9. Clinical outcomes after sensitivity analysis (CVP $>15 \mathrm{mmHg}$ ). Figure $\mathbf{S 1}$. Distribution of time to initial CVP measurements. Figure S2. Standardized mean difference (SMD) of variables before and after propensity score matching and weighting.

\section{Acknowledgements}

None

\section{Authors' contributions}

Hui Chen, Zhu Zhu and Chenyan Zhao contributed equally to this work. Jun Jin and Yao Wei conceptualized the research aims, planned the analyses and guided the literature review. Hui Chen extracted the data from the MIMIC-III database. Chenyan Zhao and Zhu Zhu participated in processing the data and doing the statistical analysis. Hui Chen wrote the first draft of the paper and the other authors provided comments and approved the final manuscript. The author(s) read and approved the final manuscript.

\section{Funding}

The protocol was financially supported by the National Natural Science Foundation of China (Grant No: 81801886).

\section{Availability of data and materials}

The datasets presented in the current study are available in the MIMIC III database (https://physionet.org/works/MIMICIIIClinicalDatabase/files/).

\section{Ethics approval and consent to participate}

The establishment of this database was approved by the Massachusetts Institute of Technology (Cambridge, MA) and Beth Israel Deaconess Medical Center (Boston, MA), and consent was obtained for the original data collection. Therefore, the ethical approval statement and the need for informed consent were waived for this manuscript.

\section{Consent for publication}

Not applicable

\section{Competing interests}

The authors declare that they have no competing interests.

\section{Author details}

'Department of Critical Care Medicine, The First Affiliated Hospital of Soochow University, Suzhou 215000, Jiangsu, China. ${ }^{2}$ Department of General Surgery, The Affiliated Suzhou Science \& Technology Town Hospital of Nanjing Medical University, Suzhou 215000, Jiangsu, China. ${ }^{3}$ Department of Intensive Care Medicine, Yancheng City No.1 People's Hospital, Yancheng 224000, Jiangsu, China. ${ }^{4}$ Department of Intensive Care Medicine, The First Affiliated Hospital of Soochow University, No. 899 Pinghai Road, Suzhou 215000, Jiangsu, China.

\section{Received: 17 February 2020 Accepted: 26 June 2020}

\section{Published online: 14 July 2020}

\section{References}

1. Machado FR, Cavalcanti AB, Bozza FA, Ferreira EM, Angotti Carrara FS, Sousa J, Caixeta N, Salomao R, Angus DC, Pontes Azevedo LC. The epidemiology of sepsis in Brazilian intensive care units (the Sepsis PREvalence Assessment Database, SPREAD): an observational study. Lancet Infect Dis. 2017:17(11): 1180-9.
2. Liu V, Escobar GJ, Greene JD, Soule J, Whippy A, Angus DC, Iwashyna TJ. Hospital deaths in patients with sepsis from 2 independent cohorts. Jama. 2014;312(1):90-2.

3. Rivers E, Nguyen B, Havstad S, Ressler J, Muzzin A, Knoblich B, Peterson E, Tomlanovich $\mathrm{M}$. Early goal-directed therapy in the treatment of severe sepsis and septic shock. N Engl J Med. 2001;345(19):1368-77.

4. Rhodes A, Evans LE, Alhazzani W, Levy MM, Antonelli M, Ferrer R, Kumar A, Sevransky JE, Sprung CL, Nunnally ME, et al. Surviving Sepsis Campaign: International Guidelines for Management of Sepsis and Septic Shock: 2016. Intensive Care Med. 2017:43(3):304-77.

5. Biais M, Ehrmann S, Mari A, Conte B, Mahjoub Y, Desebbe O, Pottecher J, Lakhal K, Benzekri-Lefevre D, Molinari N, et al. Clinical relevance of pulse pressure variations for predicting fluid responsiveness in mechanically ventilated intensive care unit patients: the grey zone approach. Critical care (London, England). 2014;18(6):587.

6. Legrand M, Dupuis C, Simon C, Gayat E, Mateo J, Lukaszewicz AC, Payen D. Association between systemic hemodynamics and septic acute kidney injury in critically ill patients: a retrospective observational study. Critical care (London, England). 2013;17(6):R278.

7. Smyrniotis V, Kostopanagiotou G, Theodoraki K, Tsantoulas D, Contis JC. The role of central venous pressure and type of vascular control in blood loss during major liver resections. Am J Surg. 2004;187(3):398-402.

8. Magder S. Right atrial pressure in the critically ill: how to measure, what is the value, what are the limitations? Chest. 2017;151(4):908-16.

9. Venn R, Steele A, Richardson P, Poloniecki J, Grounds M, Newman P. Randomized controlled trial to investigate influence of the fluid challenge on duration of hospital stay and perioperative morbidity in patients with hip fractures. Br J Anaesth. 2002;88(1):65-71.

10. Lin SM, Huang CD, Lin HC, Liu CY, Wang CH, Kuo HP. A modified goaldirected protocol improves clinical outcomes in intensive care unit patients with septic shock: a randomized controlled trial. Shock (Augusta, Ga). 2006; 26(6):551-7.

11. Vincent JL, De Backer D. Circulatory shock. N Engl J Med. 2013;369(18):1726-34

12. Jansen TC, van Bommel J, Schoonderbeek FJ, Sleeswijk Visser SJ, van der Klooster JM, Lima AP, Willemsen SP, Bakker J. Early lactate-guided therapy in intensive care unit patients: a multicenter, open-label, randomized controlled trial. Am J Respir Crit Care Med. 2010;182(6):752-61.

13. Zhang Z, Zheng C, Kim C, Van Poucke S, Lin S, Lan P. Causal mediation analysis in the context of clinical research. Ann Transl Med. 2016;4(21):425.

14. Johnson AE, Pollard TJ, Shen L, Lehman LW, Feng M, Ghassemi M, Moody B, Szolovits P, Celi LA, Mark RG. MIMIC-III, a freely accessible critical care database. Scientific Data. 2016:3:160035.

15. Benchimol El, Smeeth L, Guttmann A, Harron K, Moher D, Petersen I, Sorensen HT, von Elm E, Langan SM. The REporting of studies Conducted using Observational Routinely-collected health Data (RECORD) statement. PLoS Med. 2015;12(10):e1001885.

16. Singer M, Deutschman CS, Seymour CW, Shankar-Hari M, Annane D, Bauer M, Bellomo R, Bernard GR, Chiche JD, Coopersmith CM, et al. The third international consensus definitions for sepsis and septic shock (Sepsis-3). Jama. 2016;315(8):801-10.

17. Zhang Z. Propensity score method: a non-parametric technique to reduce model dependence. Ann Transl Med. 2017;5(1):7.

18. Graffeo N, Latouche A, Le Tourneau C. Chevret S: ipcwswitch: an R package for inverse probability of censoring weighting with an application to switches in clinical trials. Comput Biol Med. 2019;111:103339.

19. Rowan KM, Angus DC, Bailey M, Barnato AE, Bellomo R, Canter RR, Coats TJ, Delaney A, Gimbel E, Grieve RD, et al. Early, goal-directed therapy for septic shock - a patient-level meta-analysis. N Engl J Med. 2017;376(23):2223-34.

20. Yealy DM, Kellum JA, Huang DT, Barnato AE, Weissfeld LA, Pike F, Terndrup T, Wang HE, Hou PC, LoVecchio F, et al. A randomized trial of protocolbased care for early septic shock. N Engl J Med. 2014;370(18):1683-93.

21. Hughes RE, Magovern GJ. The relationship between right atrial pressure and blood volume. AMA Archives Surg. 1959;79(2):238-43.

22. De Backer D, Vincent JL. Should we measure the central venous pressure to guide fluid management? Ten answers to 10 questions. Critical Care (London, England). 2018;22(1):43.

23. Eskesen TG, Wetterslev M, Perner A. Systematic review including re-analyses of 1148 individual data sets of central venous pressure as a predictor of fluid responsiveness. Intensive Care Med. 2016;42(3):324-32.

24. Cecconi M, De Backer D, Antonelli M, Beale R, Bakker J, Hofer C, Jaeschke R, Mebazaa A, Pinsky MR, Teboul JL, et al. Consensus on circulatory shock and 
hemodynamic monitoring. Task force of the European Society of Intensive Care Medicine. Intensive Care Med. 2014;40(12):1795-815.

25. Cecconi M, Hernandez G, Dunser M, Antonelli M, Baker T, Bakker J,

Duranteau J, Einav S, Groeneveld ABJ, Harris T, et al. Fluid administration for acute circulatory dysfunction using basic monitoring: narrative review and expert panel recommendations from an ESICM task force. Intensive Care Med. 2019;45(1):21-32

26. Boyd JH, Forbes J, Nakada TA, Walley KR, Russell JA. Fluid resuscitation in septic shock: a positive fluid balance and elevated central venous pressure are associated with increased mortality. Crit Care Med. 2011;39(2):259-65.

27. Infectious Diseases Society of America (IDSA) position statement. Why IDSA did not endorse the surviving sepsis campaign guidelines. Clin Infectious Dis. 2018;66(10):1631-5.

28. Suetrong B, Walley KR. Lactic acidosis in sepsis: It's not all anaerobic: implications for diagnosis and management. Chest. 2016;149(1):252-61.

\section{Publisher's Note}

Springer Nature remains neutral with regard to jurisdictional claims in published maps and institutional affiliations.

Ready to submit your research? Choose BMC and benefit from:

- fast, convenient online submission

- thorough peer review by experienced researchers in your field

- rapid publication on acceptance

- support for research data, including large and complex data types

- gold Open Access which fosters wider collaboration and increased citations

- maximum visibility for your research: over $100 \mathrm{M}$ website views per year

At $\mathrm{BMC}$, research is always in progress.

Learn more biomedcentral.com/submissions 